\title{
A country-wide malaria survey in Mozambique. II. Malaria attributable proportion of fever and establishment of malaria case definition in children across different epidemiological settings Samuel Mabunda*1,2, John J Aponte ${ }^{3,4}$, Armindo Tiago ${ }^{5}$ and Pedro Alonso ${ }^{3,4}$
}

\begin{abstract}
Address: ${ }^{1}$ National Malaria Control Programme, Maputo, Mozambique, ${ }^{2}$ National Institute of Health, Maputo, Mozambique, ${ }^{3}$ Centre for International Health, Hospital Clinic, Institut d'Investigacions Biomedicas August Pi i Sunyer (IDIBAPS), University of Barcelona, Barcelona, Spain, ${ }^{4}$ Centro de Investigação em Saúde da Manhiça, Maputo, Mozambique and ${ }^{5}$ Faculdade de Medicina, Departamento de Fisiologia, Universidade Eduardo Mondlane, Maputo, Mozambique
\end{abstract}

Email: Samuel Mabunda* - sjamabunda@gmail.com; John J Aponte - john.aponte@cresib.cat; Armindo Tiago - artiago99@yahoo.com.br; Pedro Alonso - palonso@clinic.ub.es

* Corresponding author

\section{Published: 2I April 2009}

Malaria Journal 2009, 8:74 doi:10.1186/1475-2875-8-74
Received: 28 July 2008

Accepted: 21 April 2009

This article is available from: http://www.malariajournal.com/content/8/I/74

(c) 2009 Mabunda et al; licensee BioMed Central Ltd.

This is an Open Access article distributed under the terms of the Creative Commons Attribution License (http://creativecommons.org/licenses/by/2.0), which permits unrestricted use, distribution, and reproduction in any medium, provided the original work is properly cited.

\begin{abstract}
Background: Protection against clinical malaria episodes is acquired slowly after frequent exposure to malaria parasites. This is reflected by a decrease with increasing age in both parasite density and incidence of clinical episodes. In many settings of stable malaria transmission, the presence of asymptomatic malaria parasite carriers is common and the definition of clinical malaria remains uncertain.
\end{abstract}

Methods: Between February 2002 and April 2003, a country-wide malaria survey was conducted in 24 districts of Mozambique, aiming to characterize the malaria transmission intensities and to estimate the proportion of fever cases attributable to malaria infections in order to establish the malaria case definition. A total of 8,816 children less than ten years of age were selected for the study. Axillary temperature was measured in all participating subjects and finger prick blood collections were taken to prepare thick and thin films for identification of parasite species and determination of parasite density. The proportion of fever cases attributable to malaria infection was estimated using a logistic regression of the fever on a monotonic function of the parasite density and, using bootstrap facilities, bootstrapped estimated confidence intervals, as well as the sensitivity and specificity for different parasite density cut-offs were produced.

Results: Overall, the prevalence of Plasmodium falciparum was $52.4 \%(4,616 / 8,816)$. The prevalence of fever (axillary temperature $\geq 37.5^{\circ} \mathrm{C}$ ) was $9.4 \%(766 / 8,816)$. Fever episodes peaked among children below 12 months of life [15.I\% (206/I,5I7)]. The lowest fever prevalence of 5.9\% (67/ I,224) was recorded amongst children between five and seven years of age. Among 4,098 parasitized children, 498/4,098 (13.02\%) had fever. The prevalence of malaria infections associated with fever peaked among children in the less than twelve months age group and thereafter decreased rapidly with increasing age $(p<0.001)$. High parasite densities were significantly associated with fever $(p<0.04)$.

The proportion of fever attributed to malaria was $37.8 \%$ ( $95 \% \mathrm{Cl} 32.9 \%-42.7 \%)$. An age-specific pattern was observed with significant variations across different regions in the country. In general, among children less than 12 months of life, the proportion of fever attributed to malaria infection 
was $43.5 \%(95 \% \mathrm{Cl} 25.8 \%-61.2 \%)$, in children aged between 12 and 59 months of age was $39.6 \%$ $(95 \% \mathrm{Cl} 30.3 \%-48.9 \%)$, and among children aged between 5 and 10 years old was $21.5 \%(95 \% \mathrm{Cl}$ I I.6\%- $31.4 \%)$.

Conclusion: This study confirms that malaria remains a major cause of febrile illness during childhood. It also defines the relation between parasite density and fever and how this varies with age and region. This may help guide case definition for clinical trials of preventive tools, as well as provide definitions that may improve the precision of measurement of the burden of disease.

\section{Background}

In many malaria endemic regions, children acquire clinical immunity to malaria, and develop anti-parasitic mechanisms during the first few years of life consequent to repetitive exposures [1]. Thus, as children grow up, they develop an aptitude to lessen the density of malaria infection and to tolerate malaria parasites in the absence of fever [2]. It appears that the capability to tolerate malaria parasites increases with age, and is influenced by the intensity of malaria transmission. For this reason, clinical malaria is age-specific with regional and seasonal variations $[3,4]$. Additionally, in such areas, observation of asymptomatic malaria parasites carriers in the population is common, and detection of malaria parasites in a blood smear, does not certainly indicate a clinical malaria episode. Although the development of symptoms and/or signs of clinical malaria is complex and multi-factorial [5], in the recent years, emphasis on the relationship between fever risk and parasite density has been largely used as an entry point to define a clinical malaria episode [6].

The concept of "pyrogenic threshold" has been proposed for defining malaria episode in endemic areas [7-9]. The occurrence of fever cases due to other causes in the presence of parasitaemia, may well result in an over-diagnosis of clinical malaria. Thus, estimation of the proportion of fever cases attributable to malaria infection is crucial to establish a more concise definition of clinical malaria. Smith et al [6] proposed a methodology to estimate the attributable proportion of fever using a logistic regression of the fever on a monotonic function of the parasite density [10]. In addition, the sensitivity and specificity of specific cut-off values of parasite density can be estimated.

In Mozambique, malaria remains one of the main causes of febrile illness among children. Transmission is perennial with regional variations throughout the country. The incidence of clinical malaria established through weekly active case detection suggests that the risk of clinical malaria is highest between the age of one and three years when children experience an average of more than two episodes per year. Based on a continuous demographic surveillance system and verbal autopsies carried out in Manhiça district, the risk of clinical malaria drops sharply after the age of six [11].
Early diagnosis and prompt treatment of cases, especially in children represent the keystone strategy aiming at reducing the burden of malaria-related morbidity and mortality. However, in most rural areas, diagnosis of clinical malaria is rather presumptive based on fever or history of fever, though a positive blood film is required to confirm the diagnosis in areas where laboratory facilities exist.

This paper reports the results of a national malaria survey, carried out in Mozambique, in which the prevalence and the intensity of malaria infections, the establishment of malaria case definition and its relation to age strata across the country were determined.

\section{Methods}

A detailed description of the methods used in this survey is presented in a companion paper [12]. Briefly, a total of 8,816 children aged below ten years of age, living in households selected from 24 districts chosen randomly in the country, were eligible for the survey. Oral informed consent was obtained and a questionnaire was completed by well-trained team members. Thick and thin smears for parasitological examination and parasite density estimation were obtained during the survey. Axillary temperatures were also recorded using digital thermometers. Overall prevalence of Plasmodium falciparum infections, fever, and malaria parasite infection associated with fever were estimated in each age group for each region and stratum.

Using the method proposed by Smith et al [6], the attributable proportion of fever due to malaria using a logistic regression of the fever on a monotonic function of the parasite density was estimated. The model used was $\operatorname{logit}\left(\pi_{i}\right)=\alpha+\beta\left(x_{i}\right)^{\tau}$, and represents a logistic regression model where $\pi_{I}$ is the probability that observation $i$ with parasite density $x i$ is a fever case. $\beta\left(x_{i}\right)^{t}$ corresponds to model type 3 described by Smith $e$ t al and is a monotonic function which is more flexible than the regression on $x_{i}$ or $\log \left(x_{i}\right)$. The models were fitted using maximum likelihood. To constrain the parameter $\tau$ to be positive, the maximum likelihood were estimated for the $\log (\tau)$. Confidence intervals for the estimated attributable fraction, the $\tau$ parameter, the sensitivity and specificity were 
obtained using the bootstrap methodology [13]. Analysis were made using Stata Statistical Software (Stata Corporation, College Station, Tx USA).

\section{Results \\ Prevalence of fever and fever associated with malaria parasites}

Overall, fever prevalence (axillary temperature $\geq 37.5^{\circ} \mathrm{C}$ ) among children was $9.4 \%(766 / 8,816)$. Table 1 , depicts the prevalence of parasite infection, fever and fever associated with parasite in different age groups. The prevalence of fever decreased rapidly with increasing age, peaking among children during the first 12 months of life [15.1\% $(206 / 1,517)]$. The lowest fever prevalence of 5.9\% (67/ $1,224)$ was recorded among children in the five to seven years of age group. Overall, the prevalence of fever associated with $P$. falciparum infections (asexual forms) accounted for 5.7\% $(498 / 8,816)$, but, among febrile children, $72.4 \%$ (554/766) were infected. The prevalence of malaria infection associated with fever peaked among children during the first year of life and thereafter decreased sharply with increasing age, and the differences among age groups were statistically significant ( $\mathrm{p}<$ $0.001)$.

The risk of being febrile increased with increasing parasite density, particularly from parasite density category equal or higher than 5,000 parasites $/ \mu$ l. High $P$. falciparum parasite densities were significantly associated with fever $(\mathrm{p}<$ 0.05 ) (Table 2). According to age group, the risk of fever increased during the first 12 months of life and thereafter decreased significantly with age $(\mathrm{p}<0.0001)$. Across the study area there were significant regional variations $(\mathrm{p}<$ $0.001)$. The highest prevalence of malaria infection associated with fever was recorded in the northern region $16.6 \%(199 / 1,313)$, while in the southern region was recorded the lowest 9.9\% (59/613). Although, there were variations across strata within regions, the differences observed were not statistically significant.

The proportion of fever attributable to malaria infection The model define malaria case as any case with at least one parasite, hence sensitivity of parasite density cut-off of one parasite or more will be always $100 \%$. Table 3, illustrates the variations of the malaria attributable proportion of fever by age group across different epidemiological settings in the study area. Overall the proportion of fever attributable to malaria infection was $37.8 \%$ (95\% CI $32.9 \%-42.7 \%$ ) (Figure 1 ). The sensitivity of parasite density cut-off of one parasite or more was $100 \%$, and the specificity was 56.2 (95\% CI 54.5\% - 57.9\%). Nevertheless for parasite density cut-off of 2,500 or more parasites, the sensitivity was $75.5 \%(95 \%$ CI $71.9 \%-79.1 \%)$ and specificity was $83.3 \%$ (95\% CI $82.4 \%-84.3 \%)$. When adjusted for age group it dropped slightly to $37.5 \%$. It decreased with increasing age, and the differences between age group categories were statistically significant $(\mathrm{p}<0.05)$.

After adjusting for region, the fever proportion attributable to malaria was $36.5 \%$, decreasing from the northern to the southern region, the differences were statistically significant $(\mathrm{p}<0.05)$. When adjusted for stratum the attributable fraction of fever was $38.1 \%$, but without significant variations across strata. When adjusted for age group and region the attributable fraction of fever dropped to $36.1 \%$ and the differences observed among regions and age groups were statistically significant $(p<0.05)$. When adjusted for age group, region and stratum there were no differences statistically significant across strata, however, across regions there were differences statistically significant $(\mathrm{p}<0.05)$.

Table I: Overall point prevalence of parasite, fever and fever associated with parasite in different age groups across the study area.

\begin{tabular}{|c|c|c|c|c|}
\hline Age group & $\begin{array}{l}\text { Recorded } \\
\mathbf{N}\end{array}$ & $\begin{array}{l}\text { Parasite } \\
\text { (\%) }\end{array}$ & $\begin{array}{l}\text { Fever } \\
(\%)\end{array}$ & $\begin{array}{c}\text { Parasite and fever } \\
\text { (\%) }\end{array}$ \\
\hline$<12$ months & 1,517 & 42.2 & 15.1 & 10.6 \\
\hline $12-23$ months & 1,609 & 55.4 & 13.2 & 10.3 \\
\hline $24-59$ months & 3,515 & 51.3 & 7.1 & 4.6 \\
\hline $5-<7$ years & 1,224 & 48.1 & 5.9 & 3.2 \\
\hline $7-<10$ years & 951 & 39.3 & 5.3 & 3.3 \\
\hline Total & 8,816 & 48.6 & 9.4 & 5.7 \\
\hline $\mathrm{p}$-Value & & $<0.001$ & $<0.001$ & $<0.001$ \\
\hline
\end{tabular}


Table 2: Proportion of fever cases according to parasite density category

\begin{tabular}{cc}
\hline Parasite Density (parasites/ $\mu \mathrm{l})$ & Fever Cases (\%) \\
\hline 0 & 6.9 \\
\hline $1-499$ & 7.2 \\
\hline $500-4,999$ & 10.4 \\
\hline $5,000-49,000$ & 21.3 \\
\hline$\geq 50,000$ & 42.1 \\
\hline $\mathrm{P}-$ Value & $<0.001$ \\
\hline
\end{tabular}

\section{Among children less than twelve months of age}

The proportion of fever attributable to malaria infection among children less than 12 months of life was $43.5 \%$ (95\% CI 25.8\% - 61.2\%) (Figure 2). For parasite density cut-off of one or more parasites/ $\mu \mathrm{l}$, the specificity was $61.8 \%$ (95\% CI 58.9\% - 64.7\%). For parasite density cutoff of 2,500 or more parasites/ $\mu$ l the sensitivity was $72.9 \%$ (95\% CI $62.2 \%-83.6 \%)$, and the specificity was 83.0 (95\% CI $79.7 \%-86.4 \%)$. After adjusting for region the attributable fraction dropped to $41.4 \%$. Differences across regions were observed, but only for the central region the differences were statistically significant $(p<0.05)$. After adjusting for stratum it was $44.5 \%$. It increased from the coastal strata to the highland strata, though, there were no differences statistically significant.

\section{Among children aged between 12 and 59 months}

The proportion of fever attributable to malaria infection among children aged between 12 and 59 months of age was $39.6 \%$ (95\% CI 30.3\% - 48.9\%) (Figure 3). For para-

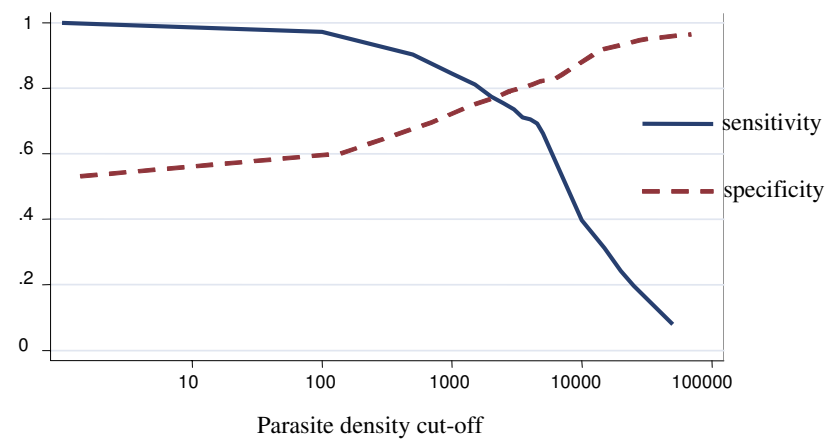

Figure I

Sensitivity and specificity curves and attributable proportion of clinical malaria among children in Mozambique. Overall country-wide, children less than 10 years of age. Attributable proportion: $37.8 \%$. site density cut-off of one or more parasites/ $\mu$ the specificity was $52.0 \%(95 \%$ CI $48.7 \%-55.4 \%)$. For parasite density cut-off of 2,500 or more parasites/ $\mu$ l the sensitivity was $77.4 \%(95 \%$ CI $72.5 \%-82.3 \%)$, and the specificity was 80.9 (95\% CI $79.9 \%-82.0 \%)$. After adjusting for region it dropped to $38.4 \%$, decreasing form the northern to the southern region. The differences observed across regions were statistically significant $(\mathrm{p}<0.05)$. When adjusted for strata it was $40.0 \%$. It increased slightly from the coastal to the highland stratum. However, the differences were not statistically significant across strata. After adjusting for age group and region was $37.8 \%$, increasing from the coastal to the highland stratum. It decreased from the northern to the southern region. There were differences statistically significant across regions. Nevertheless, after adjusting for age group and stratum there were no differences statistically significant across the strata.

\section{Among children above five years of age}

The proportion of fever attributable to malaria infection among children aged between 5 and 10 years old was $21.5 \%$ (95\% CI 11.6\% - 31.4\%) (Figure 4). For parasite density cut-off of one or more parasites/ $\mu$ l, the specificity was $61.1 \%$ (95\% CI 56.5\% - 65.6\%). For parasite density cut-off of 2,500 or more parasites/ $\mu$ l the sensitivity was $68.2 \%$ (95\% CI $45.5 \%-90.9 \%)$, and the specificity was 89.2 (95\% CI $85.1 \%$ - 93.3\%). After adjusting for region it dropped to $20.2 \%$, decreasing form the northern to the southern. The differences observed across regions were statistically significant $(\mathrm{p}<0.05)$. When adjusted for stratum it was $21.2 \%$, however, the differences observed across strata were not statistically significant.

\section{Variations across different epidemiological settings}

Overall, malaria attributable proportion of fever showed variations throughout various regions in the country, decreasing from north-to-south. Within the northern region malaria attributable proportion of fever was $37.0 \%$ (Figure 5), the highest malaria attributable proportion of fever was recorded in the centre-northern region (Figure 6), while in the central and southern region were recorded the lowest malaria attributable proportion of fever of $35.5 \%$ (Figure 7 ) and $31.1 \%$ (Figure 8 ) respectively.

\section{Discussion}

In this survey, the proportion of fever cases attributable to malaria was $37.8 \%$ (95\% CI $32.9 \%-42.7 \%)$, representing the proportion of febrile morbidity that would have been removed if malaria infections were eliminated among children in various settings in the study area. On the other hand, the results of this study highlighted the importance of other fever episodes not attributed to malaria infections in the study area.

In many malaria endemic areas, the burden of disease, a definition based on fever or reported episode of fever 
Table 3: Overall malaria attributable proportion of fever by age group across the study area.

\begin{tabular}{|c|c|c|c|}
\hline Age group & $\begin{array}{c}\text { Fever cases } \\
\mathbf{N}\end{array}$ & $\begin{array}{l}\text { Infected cases } \\
\mathbf{N}\end{array}$ & $\begin{array}{c}\text { Attributable Proportion } \\
\%(95 \% \mathrm{Cl})\end{array}$ \\
\hline$<12$ months & 206 & 641 & $43.5(25.8-61.2)$ \\
\hline $12-29$ months & 439 & 2,556 & $39.6(30.3-48.9)$ \\
\hline $5-<10$ years & 121 & 901 & $21.5(11.6-31.4)$ \\
\hline Total & 766 & 4,098 & $37.8(32.9-42.7)$ \\
\hline $\mathrm{p}$-Value & & $<0.001$ & $<0.001$ \\
\hline
\end{tabular}

within the previous 48 hours in the presence of any level of parasitaemia, would result in an over-diagnosis of malaria cases. This study was conducted during high malaria transmission season, nevertheless, among febrile children approximately one third did not have malaria parasites. This finding has significant implications on the treatment policy, particularly in rural areas where in the absence of laboratorial diagnosis, all febrile cases would be considered as clinical malaria episodes. Moreover, even among febrile parasitized children, the proportion attributable to malaria infection was less than $40 \%$.

The association between malaria infection and body temperature varies significantly among children. Despite, that the definition of clinical malaria have been related to fever episode and presence of parasites in the blood stream, in endemic-malaria areas, manifestations of clinical malaria have a wide spectrum [14] and the parasite density required to trigger fever differs significantly from one individual to another [4]. It was clear that all malaria cases had malaria parasites and fever, however, children with fever in the absence of malaria parasites are not necessarily

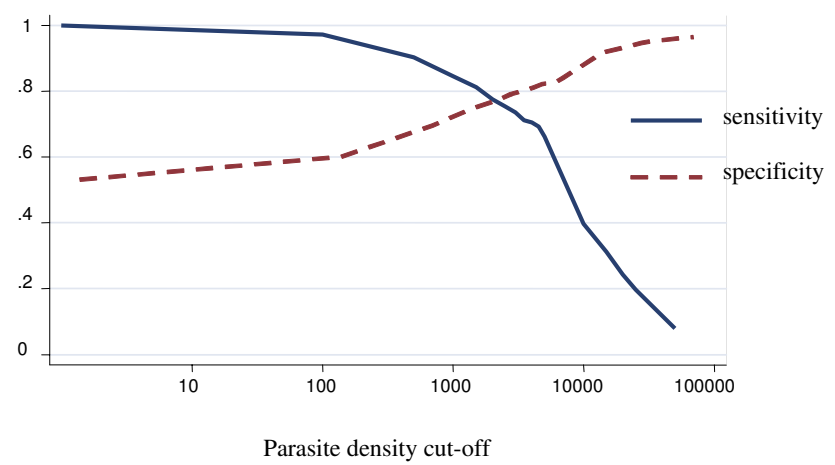

\section{Figure 2}

Sensitivity and specificity curves and attributable proportion of clinical malaria among children in Mozambique. Overall country-wide, children less than 12 months of age. Attributable proportion: $43.5 \%$. non-malaria cases, since parasitaemia is a fluctuant variable and a child with malaria may have parasitaemia at undetectable levels at a given time.

In the study area, the majority of parasitized children were asymptomatic carriers, and not all fever episodes were associated with malaria parasites, hence very few fever episodes associated with asexual $P$. falciparum infections were observed. Additionally, the risk of fever among parasitized children was age-dependent, and increased with increasing mean parasite density.

In the study area, the overall proportion of fever cases attributable to the presence of malaria infection, when adjusted for age showed significant variations. Among younger children, it was $43.5 \%$ and decreased with increasing age to reach a low of $21.5 \%$ among older children. Therefore, the proportion of fever cases attributed to parasitaemia, the sensitivity and specificity of clinical malaria definition was age-specific. Consequently, among children exposed to malaria infection, the outcome or the risk of developing fever as a clinical manifestation of malaria infection, decreased with increasing age. These

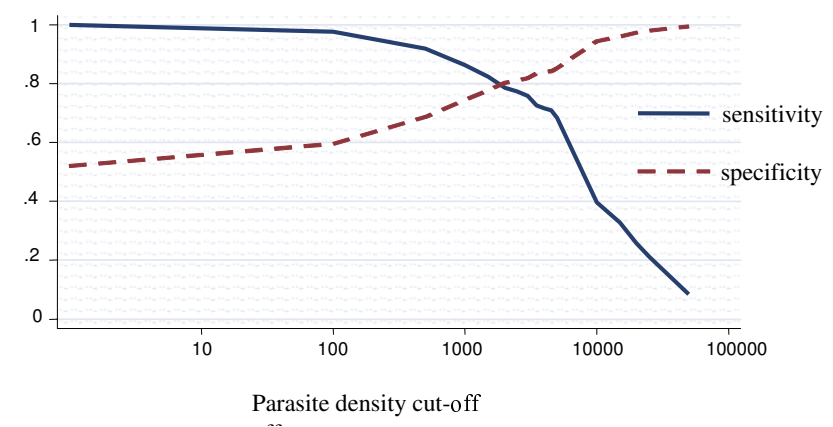

Figure 3

Sensitivity and specificity curves and attributable proportion of clinical malaria among children in Mozambique. Overall country-wide, children aged between 12 - 59 months. Attributable proportion: $39.6 \%$ 


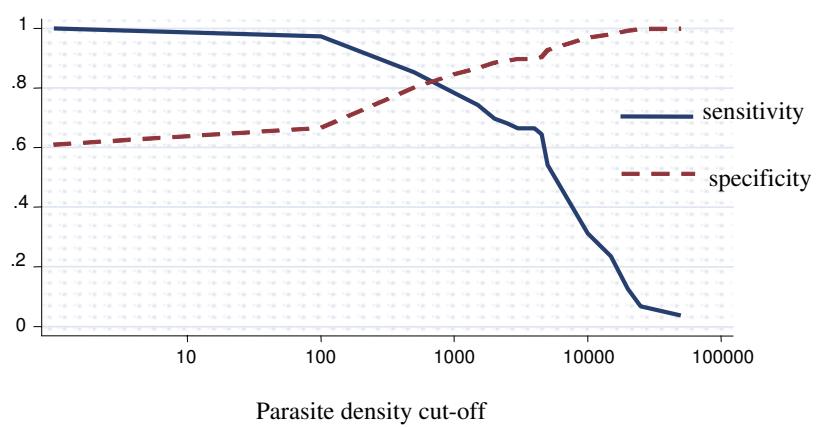

Figure 4

Sensitivity and specificity curves and attributable proportion of clinical malaria among children in Mozambique. Overall country-wide, children aged between 5 and 10 years. Attributable proportion: $21.5 \%$.

findings corroborate with results from other studies carried out in highly endemic areas $[1,3,4]$.

Regional variations on the proportion of fever attributable to malaria infection among children have been reported from other community-based surveys in endemic-malaria areas [1], highlighting the changing patterns of the relationship between malaria parasites and the host $[2,4]$. Based on the analysis of the age specific sensitivity and specificity confidence intervals for the proportion of fever attributable to malaria, the sensitivity and specificity for the cut-off-points definition for one parasite/ $\mu$ l and 2,500 parasites/ $\mu$ l were obtained for different age groups. It was crucial to determine age specific sensitivity and specificity, otherwise lack of specificity would result in a biased estimate of case definition and lack of sensitivity would result in a loss of power. However, to obtain accurate estimates of the efficacy of one intervention in clinical studies, diagnostic specificity is more important thus age-specific cutoff and region specific should be considered.

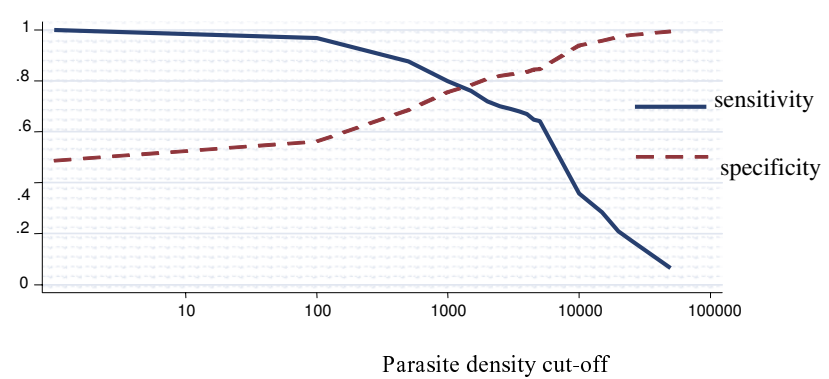

Figure 5

Sensitivity and specificity curves and attributable proportion of clinical malaria among children in Mozambique. Northern region. Attributable proportion: $37.0 \%$.

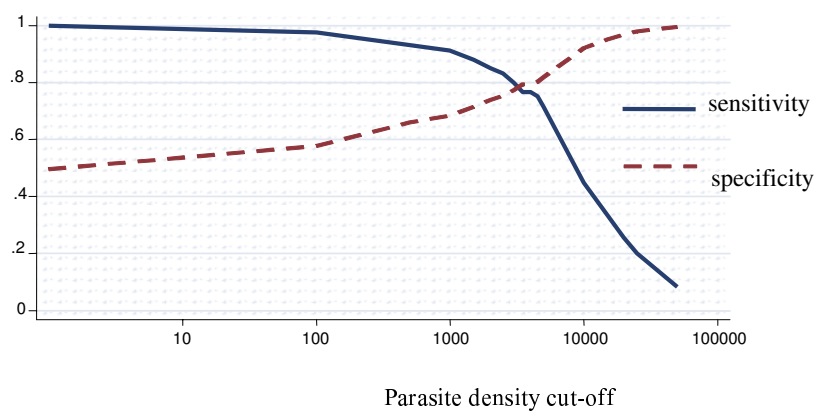

Figure 6

Sensitivity and specificity curves and attributable proportion of clinical malaria among children in Mozambique. Centre-Northern region. Attributable proportion: $48.2 \%$.

The case definitions derived from this survey, have shown the relationship between parasite density and fever and how this varies with age and region. This may help guide case definition for clinical trials of preventive tools, as well as provide definitions that may improve the precision of burden of disease assessment.

This study confirms that malaria infection remains a major cause of febrile illness during childhood. However, other causes of fever should be considered in case management of febrile illnesses during childhood.

\section{Competing interests}

The authors declare that they have no competing interests.

\section{Authors' contributions}

SM made a substantial contribution on conception and design of the study, coordinated and supervised data collection in all regions, interpretation of data, performed all

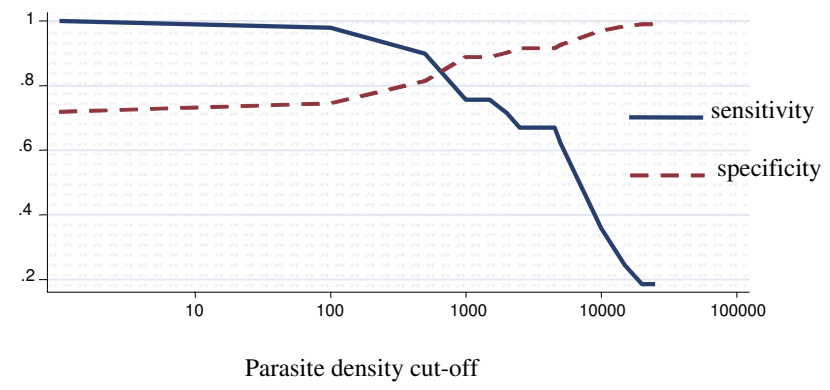

Figure 7

Sensitivity and specificity curves and attributable proportion of clinical malaria among children in Mozambique. Central region. Attributable proportion: $35.6 \%$. 


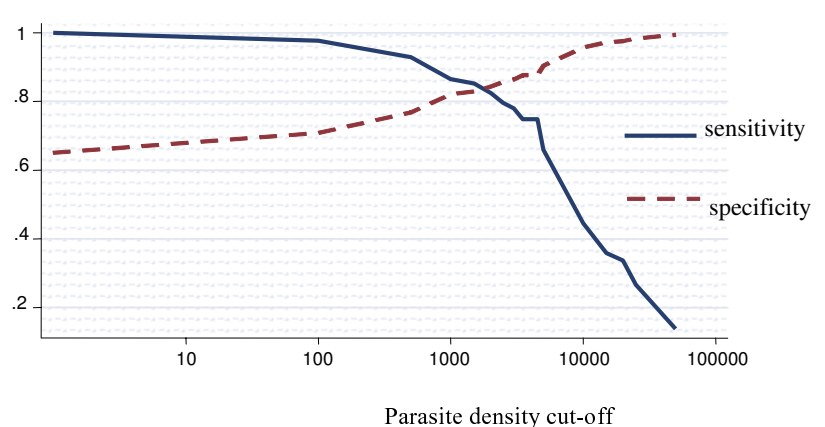

Figure 8

Sensitivity and specificity curves and attributable proportion of clinical malaria among children in Mozambique. Southern region. Attributable proportion: $31.1 \%$.

statistical analysis and wrote the manuscript. JA gave a major contribution on data interpretation and statistical analysis and helped to draft and revised the manuscript. AT helped to draft, gave contribution and critically revised the manuscript. PA gave a major contribution on conception and study design and helped to draft and critically revised the manuscript. All authors read and approved the final manuscript.

\section{Acknowledgements}

We thank all mothers and parents of all children for their co-operation and participation in this study. We also thank field and laboratory staff from the National Malaria Control Program, The National Institute of Health and from Manhiça Health Research Centre.

This study was funded by the government of Mozambique, CISM, and the Spanish Agency of International Cooperation (AECl) grant.

\section{References}

I. Smith T, Charlwood JD, Kitua AY, Masanja H, Mwankusye S, Alonso PL, Tanner M: Relationships of malaria morbidity with exposure to Plasmodium falciparum in young children in a highly endemic area. Am J Trop Med Hyg 1998, 59:252-257.

2. Bloland PB, Boriga DA, Ruebush TK, McCormick JB, Roberts JM, Oloo AJ, Hawley W, Lal A, Nahalen B, Campbell CC: Longitudinal cohort study of the epidemiology of malaria infections in an area of intense malaria transmission II. Descriptive epidemiology of malaria infection and disease among children. $\mathrm{Am} J$ Trop Med Hyg 1999, 60:641-648.

3. Baird JK, Jones TR, Danudirgo EW, Annis BA, Bangs MJ, Basri H, Purnomo, Masbar S: Age-dependent acquired protection against Plasmodium falciparum in people having two years exposure to hyperendemic malaria. Am J Trop Med Hyg I99I, 45:65-76.

4. Rogier C, Commenges D, Trape JP: Evidence for an age-dependent pyrogenic threshold of Plasmodium falciparum parasitaemia in highly endemic populations. Am J Trop Med Hyg 1996, 54:613-619.

5. Greenwood BM, Marsh K, Snow RW: Why do some African children develop severe malaria? Parasitol Today |99|, 7:277-281.

6. Smith T, Armstrong-Schellenberg JRM, Hayes RC: Attributable fraction estimates and case definitions for malaria in endemic areas. Statistical Medine 1994, 13:2345-2358.
7. Greenwood BM, Bradley AK, Greenwood AM, Byass P, Jammeh K, Marsh K, Tulloch S, Oldfield FSJ, Hayes R: Mortality and morbidity from malaria among children in a rural area of The Gambia, West Africa. Trans R Soc Trop Med Hyg 1987, 8 I:478-486.

8. Rougemont A, Breslow N, Brenner E, Moret AL, Dumbo O, Dolo A, Soula G, Perrin L: Epidemiological basis for clinical diagnosis of childhood malaria in endemic zone in West Africa. Lancet |991, 338:1292-1295.

9. Armstrong-Schellenberg JRM, Smith T, Alonso PL, Hayes RJ: What is clinical malaria? Finding case definitions for field research in highly endemic areas. Parasitol Today 1994, 10:439-442.

10. McGuinness D, Koram K, Bennett S, Wagner G, Nkrumah F, Riley E: Clinical case definitions for malaria: clinical malaria associated with very low parasite densities in African infants. Trans $R$ Soc Trop Med Hyg 1998, 92:527-3I.

II. Alonso PL, Saúte F, Aponte JJ, Gómez-Olivé FX, Nhacolo A, Thomson R, Macete E, Abacassamo F, Ventura PJ, Bosch X, Menéndez C, Dgedge M: Manhiça DSS, Mozambique. In Population and Health in Developing Countries Volume I. Population, Health, and Survival at INDEPTH Sites. Ist edition. Edited by: INDEPTH. Ottawa, International Development Research Centre (IDRC); 2002:189-195.

12. Mabunda S, Casimiro S, Quinto L, Alonso P: A country wide malaria survey in Mozambique I. Plasmodium falciparum infection in children across different epidemiological settings. Malar J 2008, 7:216.

13. Efron Bradley, Tibshirani Robert J: An introduction to the Bootstrap. Monographs on Statistics and Applied Probability 57. Chapman \& Hall, London UK; 1993.

14. Bruce-Chwatt L: Malaria and its control: present situation and future prospects. Annu Rev Public Health 1987, 8:75-I I0.

Publish with Bio Med Central and every scientist can read your work free of charge

"BioMed Central will be the most significant development for disseminating the results of biomedical research in our lifetime. "

Sir Paul Nurse, Cancer Research UK

Your research papers will be:

- available free of charge to the entire biomedical community

- peer reviewed and published immediately upon acceptance

- cited in PubMed and archived on PubMed Central

- yours - you keep the copyright
BioMedcentral 